\title{
Ranks of a Constrained Hermitian Matrix Expression with Applications
}

\author{
Shao-Wen Yu \\ Department of Mathematics, East China University of Science and Technology, Shanghai 200237, China \\ Correspondence should be addressed to Shao-Wen Yu; yushaowen@ecust.edu.cn
}

Received 12 November 2012; Accepted 4 January 2013

Academic Editor: Yang Zhang

Copyright (C) 2013 Shao-Wen Yu. This is an open access article distributed under the Creative Commons Attribution License, which permits unrestricted use, distribution, and reproduction in any medium, provided the original work is properly cited.

We establish the formulas of the maximal and minimal ranks of the quaternion Hermitian matrix expression $C_{4}-A_{4} X A_{4}^{*}$ where $X$ is a Hermitian solution to quaternion matrix equations $A_{1} X=C_{1}, X B_{1}=C_{2}$, and $A_{3} X A_{3}^{*}=C_{3}$. As applications, we give a new necessary and sufficient condition for the existence of Hermitian solution to the system of matrix equations $A_{1} X=C_{1}, X B_{1}=C_{2}$, $A_{3} X A_{3}^{*}=C_{3}$, and $A_{4} X A_{4}^{*}=C_{4}$, which was investigated by Wang and Wu, 2010, by rank equalities. In addition, extremal ranks of the generalized Hermitian Schur complement $C_{4}-A_{4} A_{3}^{\sim} A_{4}^{*}$ with respect to a Hermitian g-inverse $A_{3}^{\sim}$ of $A_{3}$, which is a common solution to quaternion matrix equations $A_{1} X=C_{1}$ and $X B_{1}=C_{2}$, are also considered.

\section{Introduction}

Throughout this paper, we denote the real number field by $\mathbb{R}$, the complex number field by $\mathbb{C}$, the set of all $m \times n$ matrices over the quaternion algebra

$$
\begin{aligned}
\mathbb{W}=\left\{a_{0}+a_{1} i+a_{2} j+a_{3} k \mid i^{2}=j^{2}\right. \\
\left.=k^{2}=i j k=-1, a_{0}, a_{1}, a_{2}, a_{3} \in \mathbb{R}\right\}
\end{aligned}
$$

by $\mathbb{Q}^{m \times n}$, the identity matrix with the appropriate size by $I$, the column right space, the row left space of a matrix $A$ over $\mathbb{U}$ by $\mathscr{R}(A), \mathscr{N}(A)$, respectively, the dimension of $\mathscr{R}(A)$ by $\operatorname{dim} \mathscr{R}(A)$, a Hermitian g-inverse of a matrix $A$ by $X=A^{-}$ which satisfies $A A^{\wedge} A=A$ and $X=X^{*}$, and the MoorePenrose inverse of matrix $A$ over $\llbracket$ by $A^{\dagger}$ which satisfies four Penrose equations $A A^{\dagger} A=A, A^{\dagger} A A^{\dagger}=A^{\dagger},\left(A A^{\dagger}\right)^{*}=$ $A A^{\dagger}$, and $\left(A^{\dagger} A\right)^{*}=A^{\dagger} A$. In this case $A^{\dagger}$ is unique and $\left(A^{\dagger}\right)^{*}=\left(A^{*}\right)^{\dagger}$. Moreover, $R_{A}$ and $L_{A}$ stand for the two projectors $L_{A}=I-A^{\dagger} A, R_{A}=I-A A^{\dagger}$ induced by $A$. Clearly, $R_{A}$ and $L_{A}$ are idempotent, Hermitian and $R_{A}=L_{A^{*}}$. By [1], for a quaternion matrix $A, \operatorname{dim} \mathscr{R}(A)=\operatorname{dim} \mathscr{N}(A)$. $\operatorname{dim} \mathscr{R}(A)$ is called the rank of a quaternion matrix $A$ and denoted by $r(A)$.
Mitra [2] investigated the system of matrix equations

$$
A_{1} X=C_{1}, \quad X B_{1}=C_{2} .
$$

Khatri and Mitra [3] gave necessary and sufficient conditions for the existence of the common Hermitian solution to (2) and presented an explicit expression for the general Hermitian solution to (2) by generalized inverses. Using the singular value decomposition (SVD), Yuan [4] investigated the general symmetric solution of (2) over the real number field $\mathbb{R}$. By the SVD, Dai and Lancaster [5] considered the symmetric solution of equation

$$
A X A^{*}=C
$$

over $\mathbb{R}$, which was motivated and illustrated with an inverse problem of vibration theory. Groß [6], Tian and Liu [7] gave the solvability conditions for Hermitian solution and its expressions of (3) over $\mathbb{C}$ in terms of generalized inverses, respectively. Liu, Tian and Takane [8] investigated ranks of Hermitian and skew-Hermitian solutions to the matrix equation (3). By using the generalized SVD, Chang and Wang [9] examined the symmetric solution to the matrix equations

$$
A_{3} X A_{3}^{*}=C_{3}, \quad A_{4} X A_{4}^{*}=C_{4}
$$


over $\mathbb{R}$. Note that all the matrix equations mentioned above are special cases of

$$
\begin{aligned}
& A_{1} X=C_{1}, \quad X B_{1}=C_{2}, \\
& A_{3} X A_{3}^{*}=C_{3}, \quad A_{4} X A_{4}^{*}=C_{4} .
\end{aligned}
$$

Wang and $\mathrm{Wu}[10]$ gave some necessary and sufficient conditions for the existence of the common Hermitian solution to (5) for operators between Hilbert $\mathrm{C}^{*}$-modules by generalized inverses and range inclusion of matrices. In view of the complicated computations of the generalized inverses of matrices, we naturally hope to establish a more practical, necessary, and sufficient condition for system (5) over quaternion algebra to have Hermitian solution by rank equalities.

As is known to us, solutions to matrix equations and ranks of solutions to matrix equations have been considered previously by many authors [10-34], and extremal ranks of matrix expressions can be used to characterize their rank invariance, nonsingularity, range inclusion, and solvability conditions of matrix equations. Tian and Cheng [35] investigated the maximal and minimal ranks of $A-B X C$ with respect to $X$ with applications; Tian [36] gave the maximal and minimal ranks of $A_{1}-B_{1} X C_{1}$ subject to a consistent matrix equation $B_{2} X C_{2}=A_{2}$. Tian and Liu [7] established the solvability conditions for (4) to have a Hermitian solution over $\mathbb{C}$ by the ranks of coefficient matrices. Wang and Jiang [20] derived extreme ranks of (skew)Hermitian solutions to a quaternion matrix equation $A X A^{*}+B Y B^{*}=C$. Wang, Yu and Lin [31] derived the extremal ranks of $C_{4}-A_{4} X B_{4}$ subject to a consistent system of matrix equations

$$
A_{1} X=C_{1}, \quad X B_{1}=C_{2}, \quad A_{3} X B_{3}=C_{3}
$$

over $\mathbb{H}$ and gave a new solvability condition to system

$$
\begin{aligned}
& A_{1} X=C_{1}, \quad X B_{1}=C_{2}, \\
& A_{3} X B_{3}=C_{3}, \quad A_{4} X B_{4}=C_{4} .
\end{aligned}
$$

In matrix theory and its applications, there are many matrix expressions that have symmetric patterns or involve Hermitian (skew-Hermitian) matrices. For example,

$$
\begin{aligned}
& A-B X B^{*}, \quad A-B X \pm X^{*} B^{*}, \\
& A-B X B^{*}-C Y C^{*}, \quad A-B X C \pm(B X C)^{*},
\end{aligned}
$$

where $A= \pm A^{*}, B$, and $C$ are given and $X$ and $Y$ are variable matrices. In recent papers $[7,8,37,38]$, Liu and Tian considered some maximization and minimization problems on the ranks of Hermitian matrix expressions (8).

Define a Hermitian matrix expression

$$
f(X)=C_{4}-A_{4} X A_{4}^{*},
$$

where $C_{4}=C_{4}^{*}$; we have an observation that by investigating extremal ranks of (9), where $X$ is a Hermitian solution to a system of matrix equations

$$
A_{1} X=C_{1}, \quad X B_{1}=C_{2}, \quad A_{3} X A_{3}^{*}=C_{3} .
$$

A new necessary and sufficient condition for system (5) to have Hermitian solution can be given by rank equalities, which is more practical than one given by generalized inverses and range inclusion of matrices.

It is well known that Schur complement is one of the most important matrix expressions in matrix theory; there have been many results in the literature on Schur complements and their applications [39-41]. Tian [36, 42] has investigated the maximal and minimal ranks of Schur complements with applications.

Motivated by the work mentioned above, we in this paper investigate the extremal ranks of the quaternion Hermitian matrix expression (9) subject to the consistent system of quaternion matrix equations (10) and its applications. In Section 2, we derive the formulas of extremal ranks of (9) with respect to Hermitian solution of (10). As applications, in Section 3, we give a new, necessary, and sufficient condition for the existence of Hermitian solution to system (5) by rank equalities. In Section 4, we derive extremal ranks of generalized Hermitian Schur complement subject to (2). We also consider the rank invariance problem in Section 5.

\section{Extremal Ranks of (9) Subject to System (10)}

Corollary 8 in [10] over Hilbert $C^{*}$-modules can be changed into the following lemma over $\mathbb{\sharp}$.

Lemma 1. Let $A_{1}, C_{1} \in \mathbb{Q}^{m \times n}, B_{1}, C_{2} \in \mathbb{T}^{n \times s}, A_{3} \in$ $\mathbb{Q} \mathbb{Q}^{r \times n}, C_{3} \in \mathbb{Q}^{r \times r}$ be given, and $F=B_{1}^{*} L_{A_{1}}, M=S L_{F}, S=$ $A_{3} L_{A_{1}}, D=C_{2}^{*}-B_{1}^{*} A_{1}^{\dagger} C_{1}, J=A_{1}^{\dagger} C_{1}+F^{\dagger} D, G=C_{3}-A_{3}(J+$ $\left.L_{A_{1}} L_{F}^{*} J^{*}\right) A_{3}^{*}$; then the following statements are equivalent:

(1) the system (10) have a Hermitian solution,

(2) $C_{3}=C_{3}^{*}$,

$$
\begin{aligned}
& A_{1} C_{2}=C_{1} B_{1}, \quad A_{1} C_{1}^{*}=C_{1} A_{1}^{*}, \quad B_{1}^{*} C_{2}=C_{2}^{*} B_{1} \text {, } \\
& R_{A_{1}} C_{1}=0, \quad R_{F} D=0, \quad R_{M} G=0,
\end{aligned}
$$

(3) $C_{3}=C_{3}^{*}$; the equalities in (11) hold and

$$
\begin{aligned}
& r\left[\begin{array}{ll}
A_{1} & C_{1}
\end{array}\right]=r\left(A_{1}\right), \quad r\left[\begin{array}{ll}
A_{1} & C_{1} \\
B_{1}^{*} & C_{2}^{*}
\end{array}\right]=r\left[\begin{array}{l}
A_{1} \\
B_{1}^{*}
\end{array}\right], \\
& r\left[\begin{array}{cc}
A_{1} & C_{1} A_{3}^{*} \\
B_{1}^{*} & C_{2}^{*} A_{3}^{*} \\
A_{3} & C_{3}
\end{array}\right]=r\left[\begin{array}{c}
A_{1} \\
B_{1}^{*} \\
A_{3}
\end{array}\right] \text {. }
\end{aligned}
$$

In that case, the general Hermitian solution of (10) can be expressed as

$$
\begin{aligned}
X= & J+L_{A_{1}} L_{F} J^{*}+L_{A_{1}} L_{F} M^{\dagger} G\left(M^{\dagger}\right)^{*} L_{F} L_{A_{1}} \\
& +L_{A_{1}} L_{F} L_{M} V L_{F} L_{A_{1}}+L_{A_{1}} L_{F} V^{*} L_{M} L_{F} L_{A_{1}},
\end{aligned}
$$

where $V$ is Hermitian matrix over $\mathbb{W}$ with compatible size. 
Lemma 2 (see Lemma 2.4 in [24]). Let $A \in \mathbb{T}^{m \times n}, B \in$

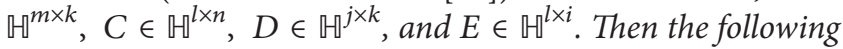
rank equalities hold:

(a) $r\left(C L_{A}\right)=r\left[{ }_{C}^{A}\right]-r(A)$,

(b) $r\left[\begin{array}{ll}B & A L_{C}\end{array}\right]=r\left[\begin{array}{ll}B & A \\ 0 & C\end{array}\right]-r(C)$,

(c) $r\left[\begin{array}{c}C \\ R_{B} A\end{array}\right]=r\left[\begin{array}{ll}C & 0 \\ A & B\end{array}\right]-r(B)$,

(d) $r\left[\begin{array}{cc}A & B L_{D} \\ R_{E} C & 0\end{array}\right]=r\left[\begin{array}{ccc}A & B & 0 \\ C & 0 & E \\ 0 & D & 0\end{array}\right]-r(D)-r(E)$.

Lemma 2 plays an important role in simplifying ranks of various block matrices.

Liu and Tian [38] has given the following lemma over a field. The result can be generalized to $\mathbb{H}$.

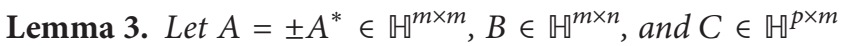
be given; then

$$
\begin{aligned}
& \max _{X \in \mathbb{H}^{n \times p}} r {\left[A-B X C \mp(B X C)^{*}\right] } \\
&= \min \left\{r\left[\begin{array}{lll}
A & B & C^{*}
\end{array}\right], r\left[\begin{array}{cc}
A & B \\
B^{*} & 0
\end{array}\right], r\left[\begin{array}{cc}
A & C^{*} \\
C & 0
\end{array}\right]\right\}, \\
& \min _{X \in \mathbb{H}^{n \times p}} r\left[\begin{array}{ll}
A-B X C \mp(B X C)^{*}
\end{array}\right] \\
&=2 r\left[\begin{array}{lll}
A & B & C^{*}
\end{array}\right]+\max \left\{s_{1}, s_{2}\right\},
\end{aligned}
$$

where

$$
\begin{aligned}
& s_{1}=r\left[\begin{array}{cc}
A & B \\
B^{*} & 0
\end{array}\right]-2 r\left[\begin{array}{ccc}
A & B & C^{*} \\
B^{*} & 0 & 0
\end{array}\right], \\
& s_{2}=r\left[\begin{array}{cc}
A & C^{*} \\
C & 0
\end{array}\right]-2 r\left[\begin{array}{ccc}
A & B & C^{*} \\
C & 0 & 0
\end{array}\right] .
\end{aligned}
$$

If $\mathscr{R}(B) \subseteq \mathscr{R}\left(C^{*}\right)$,

$$
\begin{aligned}
& \max _{X} r\left[A-B X C-(B X C)^{*}\right]=\min \left\{r\left[\begin{array}{ll}
A & C^{*}
\end{array}\right], r\left[\begin{array}{cc}
A & B \\
B^{*} & 0
\end{array}\right]\right\}, \\
& \max _{X} r\left[A-B X C-(B X C)^{*}\right]=\min \left\{r\left[A C^{*}\right], r\left[\begin{array}{cc}
A & B \\
B^{*} & 0
\end{array}\right]\right\} .
\end{aligned}
$$

Now we consider the extremal ranks of the matrix expression (9) subject to the consistent system (10).

Theorem 4. Let $A_{1}, C_{1}, B_{1}, C_{2}, A_{3}$, and $C_{3}$ be defined as Lemma $1, C_{4} \in \mathbb{T}^{t \times t}$, and $A_{4} \in \mathbb{Q}^{t \times n}$. Then the extremal ranks of the quaternion matrix expression $f(X)$ defined as (9) subject to system (10) are the following:

$$
\max r[f(X)]=\min \{a, b\},
$$

where

$$
\begin{aligned}
& a=r {\left[\begin{array}{cc}
C_{4} & A_{4} \\
C_{2}^{*} A_{4}^{*} & B_{1}^{*} \\
C_{1} A_{4}^{*} & A_{1}
\end{array}\right]-r\left[\begin{array}{l}
B_{1}^{*} \\
A_{1}
\end{array}\right], } \\
& b=r\left[\begin{array}{ccccc}
0 & A_{4}^{*} & A_{3}^{*} & B_{1} & A_{1}^{*} \\
A_{4} & C_{4} & 0 & 0 & 0 \\
A_{3} & 0 & -C_{3} & -A_{3} C_{2} & -A_{3} C_{1}^{*} \\
B_{1}^{*} & 0 & -C_{2}^{*} A_{3}^{*} & -C_{2}^{*} B_{1} & -C_{2}^{*} A_{1}^{*} \\
A_{1} & 0 & -C_{1} A_{3}^{*} & -C_{1} B_{1} & -C_{1} A_{1}^{*}
\end{array}\right]-2 r\left[\begin{array}{c}
A_{3} \\
B_{1}^{*} \\
A_{1}
\end{array}\right], \\
& \min r[f(X)]=2 r\left[\begin{array}{ccccc}
C_{4} & A_{4} \\
C_{2}^{*} A_{4}^{*} & B_{1}^{*} \\
C_{1} A_{4}^{*} & A_{1}
\end{array}\right] \\
&+ {\left[\begin{array}{ccccc}
0 & A_{4}^{*} & A_{3}^{*} & B_{1} \\
A_{4} & C_{4} & 0 & 0 \\
A_{3} & 0 & -C_{3} & -A_{3} C_{2} & -A_{3} C_{1}^{*} \\
B_{1}^{*} & 0 & -C_{2}^{*} A_{3}^{*} & -C_{2}^{*} B_{1} & -C_{2}^{*} A_{1}^{*} \\
A_{1} & 0 & -C_{1} A_{3}^{*} & -C_{1} B_{1} & -C_{1} A_{1}^{*}
\end{array}\right] } \\
& 2 r\left[\begin{array}{ccccc}
0 & A_{4}^{*} & B_{1} & A_{1}^{*} \\
A_{4} & C_{4} & 0 & 0 \\
A_{3} & 0 & -A_{3} C_{2} & -A_{3} C_{1}^{*} \\
B_{1}^{*} & 0 & -C_{2}^{*} B_{1} & -C_{2}^{*} A_{1}^{*} \\
A_{1} & 0 & -C_{1} B_{1} & -C_{1} A_{1}^{*}
\end{array}\right] .
\end{aligned}
$$

Proof. By Lemma 1, the general Hermitian solution of the system (10) can be expressed as

$$
\begin{aligned}
X= & J+L_{A_{1}} L_{F} J^{*}+L_{A_{1}} L_{F} M^{\dagger} G\left(M^{\dagger}\right)^{*} L_{F} L_{A_{1}} \\
& +L_{A_{1}} L_{F} L_{M} V L_{F} L_{A_{1}}+L_{A_{1}} L_{F} V^{*} L_{M} L_{F} L_{A_{1}},
\end{aligned}
$$

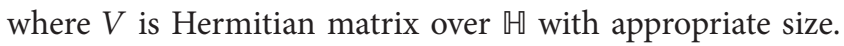
Substituting (21) into (9) yields

$$
\begin{aligned}
f(X)= & C_{4}-A_{4}\left(J+L_{A_{1}} L_{F} J^{*}\right. \\
& \left.+L_{A_{1}} L_{F} M^{\dagger} G\left(M^{\dagger}\right)^{*} L_{F} L_{A_{1}}\right) A_{4}^{*} \\
& -A_{4} L_{A_{1}} L_{F} L_{M} V L_{F} L_{A_{1}} A_{4}^{*} \\
& -A_{4} L_{A_{1}} L_{F} V^{*} L_{M} L_{F} L_{A_{1}} A_{4}^{*} .
\end{aligned}
$$


Put

$C_{4}-A_{4}\left(J+L_{A_{1}} L_{F} J^{*}+L_{A_{1}} L_{F} M^{\dagger} G\left(M^{\dagger}\right)^{*} L_{F} L_{A_{1}}\right) A_{4}^{*}=A$,

$$
\begin{aligned}
r\left[\begin{array}{cc}
A & N \\
N^{*} & 0
\end{array}\right]= & r\left[\begin{array}{ccccc}
C_{4}-A_{4} J^{\prime} A_{4}^{*} & A_{4} L_{A_{1}} L_{F} L_{M} \\
R_{M^{*}} R_{F^{*}} R_{A_{1}^{*}} A_{4}^{*} & 0
\end{array}\right] \\
=r & {\left[\begin{array}{cccccc}
C_{4}-A_{4} J^{\prime} A_{4}^{*} & A_{4} & 0 & 0 & 0 \\
A_{4}^{*} & 0 & A_{3}^{*} & B_{1} & A_{1}^{*} \\
0 & A_{3} & 0 & 0 & 0 \\
0 & B_{1}^{*} & 0 & 0 & 0 \\
0 & A_{1} & 0 & 0 & 0
\end{array}\right] } \\
& -2 r(M)-2 r(F)-2 r\left(A_{1}\right)
\end{aligned}
$$$$
J+L_{A_{1}} L_{F} J^{*}+L_{A_{1}} L_{F} M^{\dagger} G\left(M^{\dagger}\right)^{*} L_{F} L_{A_{1}}=J^{\prime},
$$

$A_{4} L_{A_{1}} L_{F} L_{M}=N$,

$L_{F} L_{A_{1}} A_{4}^{*}=P$

then

$$
f(X)=A-N V P-(N V P)^{*}
$$

Note that $A=A^{*}$ and $\mathscr{R}(N) \subseteq \mathscr{R}\left(P^{*}\right)$. Thus, applying (17) to (24), we get the following:

$$
\begin{aligned}
& \max r[f(X)]=\max _{V} r\left(A-N V P-(N V P)^{*}\right) \\
& =\min \left\{r\left[\begin{array}{ll}
A & P^{*}
\end{array}\right], r\left[\begin{array}{cc}
A & N \\
N^{*} & 0
\end{array}\right]\right\}, \\
& \min r[f(X)]=\min _{V} r\left(A-N V P-(N V P)^{*}\right) \\
& =2 r\left[\begin{array}{ll}
A & P^{*}
\end{array}\right]+r\left[\begin{array}{cc}
A & N \\
N^{*} & 0
\end{array}\right]-2 r\left[\begin{array}{cc}
A & N \\
P & 0
\end{array}\right] .
\end{aligned}
$$

Now we simplify the ranks of block matrices in (25).

In view of Lemma 2, block Gaussian elimination, (11), (12), and (23), we have the following:

$$
\begin{gathered}
r(F)=r\left(B_{1}^{*} L_{A_{1}}\right)=r\left[\begin{array}{l}
B_{1}^{*} \\
A_{1}
\end{array}\right]-r\left(A_{1}\right), \\
r(M)=r\left(S L_{F}\right)=r\left[\begin{array}{l}
S \\
F
\end{array}\right]-r(F) \\
=r\left[\begin{array}{l}
A_{3} L_{A_{1}} \\
B_{1}^{*} L_{A_{1}}
\end{array}\right]-r(F) \\
r\left[A P^{*}\right]=r\left[\begin{array}{ll}
A_{3}-A_{4} J A_{4}^{*} & \left.P^{*}\right] \\
B_{1}^{*} \\
A_{1}
\end{array}\right]-r\left(A_{1}\right)-r(F), \\
=r\left[\begin{array}{cc}
C_{4}-A_{4} J A_{4}^{*} & A_{4} L_{A_{1}} \\
0 & F
\end{array}\right]-r(F) \\
=r\left[\begin{array}{cc}
C_{4}-A_{4} J A_{4}^{*} & A_{4} \\
0 & B_{1}^{*} \\
0 & A_{1}
\end{array}\right]-r(F)-r\left(A_{1}\right) \\
=r\left[\begin{array}{cc}
C_{4} & A_{4} \\
C_{2}^{*} A_{4}^{*} & B_{1}^{*} \\
C_{1} A_{4}^{*} & A_{1}
\end{array}\right]-r\left[\begin{array}{l}
B_{1}^{*} \\
A_{1}
\end{array}\right],
\end{gathered}
$$$$
=r\left[\begin{array}{ccccc}
C_{4} & A_{4} & 0 & 0 & 0 \\
A_{4}^{*} & 0 & A_{3}^{*} & B_{1} & A_{1}^{*} \\
A_{3} J^{\prime} A_{4}^{*} & A_{3} & 0 & 0 & 0 \\
B_{1}^{*} J^{\prime} A_{4}^{*} & B_{1}^{*} & 0 & 0 & 0 \\
A_{1} J^{\prime} A_{4}^{*} & A_{1} & 0 & 0 & 0
\end{array}\right]-2 r\left[\begin{array}{c}
A_{3} \\
B_{1}^{*} \\
A_{1}
\end{array}\right]
$$$$
=r\left[\begin{array}{ccccc}
C_{4} & A_{4} & 0 & 0 & 0 \\
A_{4}^{*} & 0 & A_{3}^{*} & B_{1} & A_{1}^{*} \\
0 & A_{3} & -C_{3} & -A_{3} C_{2} & -A_{3} C_{1}^{*} \\
0 & B_{1}^{*} & -C_{2}^{*} A_{3}^{*} & -C_{2}^{*} B_{1} & -C_{2}^{*} A_{1}^{*} \\
0 & A_{1} & -C_{1} A_{3}^{*} & -C_{1} B_{1} & -C_{1} A_{1}^{*}
\end{array}\right]
$$$$
-2 r\left[\begin{array}{l}
A_{3} \\
B_{1}^{*} \\
A_{1}
\end{array}\right]
$$$$
=r\left[\begin{array}{ccccc}
0 & A_{4}^{*} & A_{3}^{*} & B_{1} & A_{1}^{*} \\
A_{4} & C_{4} & 0 & 0 & 0 \\
A_{3} & 0 & -C_{3} & -A_{3} C_{2} & -A_{3} C_{1}^{*} \\
B_{1}^{*} & 0 & -C_{2}^{*} A_{3}^{*} & -C_{2}^{*} B_{1} & -C_{2}^{*} A_{1}^{*} \\
A_{1} & 0 & -C_{1} A_{3}^{*} & -C_{1} B_{1} & -C_{1} A_{1}^{*}
\end{array}\right]
$$$$
-2 r\left[\begin{array}{l}
A_{3} \\
B_{1}^{*} \\
A_{1}
\end{array}\right]
$$$$
r\left[\begin{array}{cc}
A & N \\
P & 0
\end{array}\right]=r\left[\begin{array}{cc}
C_{4}-A_{4} J^{\prime} A_{4}^{*} & A_{4} L_{A_{1}} L_{F} L_{M} \\
R_{F^{*}} R_{A_{1}^{*}} A_{4}^{*} & 0
\end{array}\right]
$$$$
=r\left[\begin{array}{cccc}
C_{4} & A_{4} & 0 & 0 \\
A_{4}^{*} & 0 & B_{1} & A_{1}^{*} \\
0 & A_{3} & -A_{3} C_{2} & -A_{3} C_{1}^{*} \\
0 & B_{1}^{*} & -C_{2}^{*} B_{1} & -C_{2}^{*} A_{1}^{*} \\
0 & A_{1} & -C_{1} B_{1} & -C_{1} A_{1}^{*}
\end{array}\right]
$$$$
-r\left[\begin{array}{l}
A_{3} \\
B_{1}^{*} \\
A_{1}
\end{array}\right]-r\left[\begin{array}{l}
B_{1}^{*} \\
A_{1}
\end{array}\right]
$$ 


$$
\begin{aligned}
& =r\left[\begin{array}{cccc}
0 & A_{4}^{*} & B_{1} & A_{1}^{*} \\
A_{4} & C_{4} & 0 & 0 \\
A_{3} & 0 & -A_{3} C_{2} & -A_{3} C_{1}^{*} \\
B_{1}^{*} & 0 & -C_{2}^{*} B_{1} & -C_{2}^{*} A_{1}^{*} \\
A_{1} & 0 & -C_{1} B_{1} & -C_{1} A_{1}^{*}
\end{array}\right] \\
& -r\left[\begin{array}{l}
A_{3} \\
B_{1}^{*} \\
A_{1}
\end{array}\right]-r\left[\begin{array}{c}
B_{1}^{*} \\
A_{1}
\end{array}\right] .
\end{aligned}
$$

Substituting (26) into (25) yields (18) and (20).

In Theorem 4, letting $C_{4}$ vanish and $A_{4}$ be $I$ with appropriate size, respectively, we have the following.

Corollary 5. Assume that $A_{1}, C_{1} \in \mathbb{T}^{m \times n}, B_{1}, C_{2} \in$ $\mathbb{H}^{n \times s}, A_{3} \in \mathbb{T}^{r \times n}$, and $C_{3} \in \mathbb{H}^{r \times r}$ are given; then the maximal and minimal ranks of the Hermitian solution $X$ to the system (10) can be expressed as

$$
\max r(X)=\min \{a, b\}
$$

where

$$
\begin{gathered}
a=n+r\left[\begin{array}{l}
C_{2}^{*} \\
C_{1}
\end{array}\right]-r\left[\begin{array}{l}
B_{1}^{*} \\
A_{1}
\end{array}\right], \\
\min r(X)=2 r\left[\begin{array}{ccc}
C_{3} & A_{3} C_{2} & A_{3} C_{1}^{*} \\
C_{2}^{*} A_{3}^{*} & C_{2}^{*} B_{1} & C_{2}^{*} A_{1}^{*} \\
C_{1} A_{3}^{*} & C_{1} B_{1} & C_{1} A_{1}^{*}
\end{array}\right]-2 r\left[\begin{array}{l}
A_{3} \\
B_{1}^{*} \\
A_{1}
\end{array}\right], \\
+r\left[\begin{array}{cc}
C_{2}^{*} \\
C_{1}
\end{array}\right] \\
{\left[\begin{array}{ccc}
C_{2}^{*} A_{3}^{*} & C_{2}^{*} B_{1} & C_{2}^{*} A_{1}^{*} \\
C_{1} A_{3}^{*} & C_{1} B_{1} & C_{1} A_{1}^{*}
\end{array}\right]} \\
-2 r\left[\begin{array}{ll}
A_{3} C_{2} & A_{3} C_{1}^{*} \\
C_{2}^{*} B_{1} & C_{2}^{*} A_{1}^{*} \\
C_{1} B_{1} & C_{1} A_{1}^{*}
\end{array}\right] .
\end{gathered}
$$

In Theorem 4 , assuming that $A_{1}, B_{1}, C_{1}$, and $C_{2}$ vanish, we have the following.
Corollary 6. Suppose that the matrix equation $A_{3} X A_{3}^{*}=C_{3}$ is consistent; then the extremal ranks of the quaternion matrix expression $f(X)$ defined as (9) subject to $A_{3} X A_{3}^{*}=C_{3}$ are the following:

$$
\begin{aligned}
& \max r f(X)] \\
& =\min \left\{r\left[\begin{array}{ll}
C_{4} & A_{4}
\end{array}\right], r\left[\begin{array}{ccc}
0 & A_{4}^{*} & A_{3}^{*} \\
A_{4} & C_{4} & 0 \\
A_{3} & 0 & -C_{3}
\end{array}\right]-2 r\left(A_{3}\right)\right\}, \\
& \min r[f(X)]=2 r\left[\begin{array}{ll}
C_{4} & A_{4}
\end{array}\right] \\
& +r\left[\begin{array}{ccc}
0 & A_{4}^{*} & A_{3}^{*} \\
A_{4} & C_{4} & 0 \\
A_{3} & 0 & -C_{3}
\end{array}\right]-2 r\left[\begin{array}{cc}
0 & A_{4}^{*} \\
A_{4} & C_{4} \\
A_{3} & 0
\end{array}\right] .
\end{aligned}
$$

\section{A Practical Solvability Condition for Hermitian Solution to System (5)}

In this section, we use Theorem 4 to give a necessary and sufficient condition for the existence of Hermitian solution to system (5) by rank equalities.

Theorem 7. Let $A_{1}, C_{1} \in \mathbb{H}^{m \times n}, B_{1}, C_{2} \in \mathbb{H}^{n \times s}, A_{3} \in$ $\mathbb{H}^{r \times n}, C_{3} \in \mathbb{T}^{r \times r}, A_{4} \in \mathbb{T}^{t \times n}$, and $C_{4} \in \mathbb{T}^{t \times t}$ be given; then the system (5) have Hermitian solution if and only if $C_{3}=C_{3}^{*}$, (11), (13) hold, and the following equalities are all satisfied:

$$
\begin{gathered}
r\left[A_{4} C_{4}\right]=r\left(A_{4}\right), \\
r\left[\begin{array}{ccc}
C_{4} & A_{4} \\
C_{2}^{*} A_{4}^{*} & B_{1}^{*} \\
C_{1} A_{4}^{*} & A_{1}
\end{array}\right]=r\left[\begin{array}{l}
A_{4} \\
B_{1}^{*} \\
A_{1}
\end{array}\right], \\
r\left[\begin{array}{ccccc}
0 & A_{4}^{*} & A_{3}^{*} & B_{1} & A_{1}^{*} \\
A_{4} & C_{4} & 0 & 0 & 0 \\
A_{3} & 0 & -C_{3} & -A_{3} C_{2} & -A_{3} C_{1}^{*} \\
B_{1}^{*} & 0 & -C_{2}^{*} A_{3}^{*} & -C_{2}^{*} B_{1} & -C_{2}^{*} A_{1}^{*} \\
A_{1} & 0 & -C_{1} A_{3}^{*} & -C_{1} B_{1} & -C_{1} A_{1}^{*}
\end{array}\right]=2 r\left[\begin{array}{l}
A_{4} \\
A_{3} \\
B_{1}^{*} \\
A_{1}
\end{array}\right] .
\end{gathered}
$$

Proof. It is obvious that the system (5) have Hermitian solution if and only if the system (10) have Hermitian solution and

$$
\min r[f(X)]=0
$$

where $f(X)$ is defined as (9) subject to system (10). Let $X_{0}$ be a Hermitian solution to the system (5); then $X_{0}$ is a Hermitian solution to system (10) and $X_{0}$ satisfies $A_{4} X_{0} A_{4}^{*}=C_{4}$. Hence, Lemma 1 yields $C_{3}=C_{3}^{*},(11),(13)$, and (30). It follows 
from

$$
\begin{aligned}
& {\left[\begin{array}{ccccc}
I & 0 & 0 & 0 & 0 \\
0 & I & 0 & 0 & 0 \\
A_{3} X_{0} & 0 & I & 0 & 0 \\
B_{1}^{*} X_{0} & 0 & 0 & I & 0 \\
A_{1} X_{0} & 0 & 0 & 0 & I
\end{array}\right]} \\
& \times\left[\begin{array}{cccccc}
0 & A_{4}^{*} & A_{3}^{*} & B_{1} & A_{1}^{*} \\
A_{4} & C_{4} & 0 & 0 & 0 \\
A_{3} & 0 & -C_{3} & -A_{3} C_{2} & -A_{3} C_{1}^{*} \\
B_{1}^{*} & 0 & -C_{2}^{*} A_{3}^{*} & -C_{2}^{*} B_{1}-C_{2}^{*} A_{1}^{*} \\
A_{1} & 0 & -C_{1} A_{3}^{*} & -C_{1} B_{1} & -C_{1} A_{1}^{*}
\end{array}\right] \\
& \times\left[\begin{array}{ccccc}
I & -X_{0} A_{4}^{*} & 0 & 0 & 0 \\
0 & I & 0 & 0 & 0 \\
0 & 0 & I & 0 & 0 \\
0 & 0 & 0 & I & 0 \\
0 & 0 & 0 & 0 & I
\end{array}\right]=\left[\begin{array}{ccccc}
0 & A_{4}^{*} & A_{3}^{*} & B_{1} & A_{1}^{*} \\
A_{4} & 0 & 0 & 0 & 0 \\
A_{3} & 0 & 0 & 0 & 0 \\
B_{1}^{*} & 0 & 0 & 0 & 0 \\
A_{1} & 0 & 0 & 0 & 0
\end{array}\right]
\end{aligned}
$$

that (32) holds. Similarly, we can obtain (31).

Conversely, assume that $C_{3}=C_{3}^{*}$, (11), (13) hold; then by Lemma 1, system (10) have Hermitian solution. By (20), (31)(32), and

$$
r\left[\begin{array}{cccc}
0 & A_{4}^{*} & B_{1} & A_{1}^{*} \\
A_{4} & C_{4} & 0 & 0 \\
A_{3} & 0 & -A_{3} C_{2} & -A_{3} C_{1}^{*} \\
B_{1}^{*} & 0 & -C_{2}^{*} B_{1} & -C_{2}^{*} A_{1}^{*} \\
A_{1} & 0 & -C_{1} B_{1} & -C_{1} A_{1}^{*}
\end{array}\right] \geq r\left[\begin{array}{c}
A_{4} \\
A_{3} \\
B_{1}^{*} \\
A_{1}
\end{array}\right]+r\left[\begin{array}{c}
A_{4} \\
B_{1}^{*} \\
A_{1}
\end{array}\right]
$$

we can get

$$
\min r[f(X)] \leq 0
$$

However,

$$
\min r[f(X)] \geq 0
$$

Hence (33) holds, implying that the system (5) have Hermitian solution.

By Theorem 7, we can also get the following.

Corollary 8. Suppose that $A_{3}, C_{3}, A_{4}$, and $C_{4}$ are those in Theorem 7; then the quaternion matrix equations $A_{3} X A_{3}^{*}=$ $C_{3}$ and $A_{4} X A_{4}^{*}=C_{4}$ have common Hermitian solution if and only if (30) hold and the following equalities are satisfied:

$$
\begin{aligned}
r\left[A_{3}\right. & \left.C_{3}\right]=r\left(A_{3}\right), \\
r\left[\begin{array}{ccc}
0 & A_{4}^{*} & A_{3}^{*} \\
A_{4} & C_{4} & 0 \\
A_{3} & 0 & -C_{3}
\end{array}\right] & =2 r\left[\begin{array}{l}
A_{3} \\
A_{4}
\end{array}\right] .
\end{aligned}
$$

Corollary 9. Suppose that $A_{1}, C_{1} \in \mathbb{H}^{m \times n}, B_{1}, C_{2} \in \mathbb{H}^{n \times s}$, and $A, B \in \mathbb{H}^{n \times n}$ are Hermitian. Then $A$ and $B$ have a common
Hermitian g-inverse which is a solution to the system (2) if and only if (11) holds and the following equalities are all satisfied:

$$
\begin{aligned}
& r\left[\begin{array}{cc}
A_{1} & C_{1} A \\
B_{1}^{*} & C_{2}^{*} A \\
A & A
\end{array}\right]=r\left[\begin{array}{c}
A_{1} \\
B_{1}^{*} \\
A
\end{array}\right], \\
& r\left[\begin{array}{cc}
A_{1} & C_{1} B \\
B_{1}^{*} & C_{2}^{*} B \\
B & B
\end{array}\right]=r\left[\begin{array}{c}
A_{1} \\
B_{1}^{*} \\
B
\end{array}\right],
\end{aligned}
$$

$$
r\left[\begin{array}{ccccc}
0 & B & A & B_{1} & A_{1}^{*} \\
B & B & 0 & 0 & 0 \\
A & 0 & -A & -A C_{2} & -A C_{1}^{*} \\
B_{1}^{*} & 0 & -C_{2}^{*} A & -C_{2}^{*} B_{1} & -C_{2}^{*} A_{1}^{*} \\
A_{1} & 0 & -C_{1} A & -C_{1} B_{1} & -C_{1} A_{1}^{*}
\end{array}\right]=2 r\left[\begin{array}{c}
B \\
A \\
B_{1}^{*} \\
A_{1}
\end{array}\right] .
$$

\section{Extremal Ranks of Schur Complement Subject to (2)}

As is well known, for a given block matrix

$$
M=\left[\begin{array}{cc}
A & B \\
B^{*} & D
\end{array}\right],
$$

where $A$ and $D$ are Hermitian quaternion matrices with appropriate sizes, then the Hermitian Schur complement of $A$ in $M$ is defined as

$$
S_{A}=D-B^{*} A^{\sim} B,
$$

where $A^{\sim}$ is a Hermitian g-inverse of $A$, that is, $A^{\sim} \in\{X \mid$ $\left.A X A=A, X=X^{*}\right\}$.

Now we use Theorem 4 to establish the extremal ranks of $S_{A}$ given by (42) with respect to $A^{\sim}$ which is a solution to system (2).

Theorem 10. Suppose $A_{1}, C_{1} \in \mathbb{M}^{m \times n}, B_{1}, C_{2} \in \mathbb{M}^{n \times s}, D \in$ $\mathbb{H}^{t \times t}, B \in \mathbb{T}^{n \times t}$, and $A \in \mathbb{T}^{n \times n}$ are given and system (2) is consistent; then the extreme ranks of $S_{A}$ given by (42) with respect to $A^{\sim}$ which is a solution of (2) are the following:

$$
\max _{\substack{A_{1} A^{\sim}=C_{1} \\ A^{\sim} B_{1}=C_{2}}} r\left(S_{A}\right)=\min \{a, b\}
$$


where

$$
\begin{aligned}
& a=r\left[\begin{array}{cc}
D & B^{*} \\
C_{2}^{*} B & B_{1}^{*} \\
C_{1} B & A_{1}
\end{array}\right]-r\left[\begin{array}{l}
B_{1}^{*} \\
A_{1}
\end{array}\right] \\
& b=r\left[\begin{array}{ccccc}
0 & B & A & B_{1} & A_{1}^{*} \\
B^{*} & D & 0 & 0 & 0 \\
A & 0 & -A & -A C_{2} & -A C_{1}^{*} \\
B_{1}^{*} & 0 & -C_{2}^{*} A & -C_{2}^{*} B_{1} & -C_{2}^{*} A_{1}^{*} \\
A_{1} & 0 & -C_{1} A & -C_{1} B_{1} & -C_{1} A_{1}^{*}
\end{array}\right]-2 r\left[\begin{array}{c}
A \\
B_{1}^{*} \\
A_{1}
\end{array}\right], \\
& \min _{\substack{A_{1} A^{\sim}=C_{1} \\
A^{\sim} B_{1}=C_{2}}} r\left(S_{A}\right)=2 r\left[\begin{array}{cc}
D & B^{*} \\
C_{2}^{*} B & B_{1}^{*} \\
C_{1} B & A_{1}
\end{array}\right] \\
& +r\left[\begin{array}{ccccc}
0 & B & A & B_{1} & A_{1}^{*} \\
B^{*} & D & 0 & 0 & 0 \\
A & 0 & -A & -A C_{2} & -A C_{1}^{*} \\
B_{1}^{*} & 0 & -C_{2}^{*} A & -C_{2}^{*} B_{1} & -C_{2}^{*} A_{1}^{*} \\
A_{1} & 0 & -C_{1} A & -C_{1} B_{1} & -C_{1} A_{1}^{*}
\end{array}\right] \\
& -2 r\left[\begin{array}{cccc}
0 & B & B_{1} & A_{1}^{*} \\
B^{*} & D & 0 & 0 \\
A & 0 & -A C_{2} & -A C_{1}^{*} \\
B_{1}^{*} & 0 & -C_{2}^{*} B_{1} & -C_{2}^{*} A_{1}^{*} \\
A_{1} & 0 & -C_{1} B_{1} & -C_{1} A_{1}^{*}
\end{array}\right] .
\end{aligned}
$$

Proof. It is obvious that

$$
\begin{array}{rl}
\max _{A_{1} A^{\sim}=C_{1}, A^{\sim} B_{1}=C_{2}} r\left(D-B^{*} A^{\sim} B\right) & \\
& =\max _{A_{1} X=C_{1}, X B_{1}=C_{2}, A X A=A} r\left(D-B^{*} X B\right), \\
A_{1} A^{\sim}=C_{1}, A^{\sim} B_{1}=C_{2} & r\left(D-B^{*} A^{\sim} B\right) \\
& =\min _{A_{1} X=C_{1}, X B_{1}=C_{2}, A X A=A} r\left(D-B^{*} X B\right) .
\end{array}
$$

Thus in Theorem 4 and its proof, letting $A_{3}=A_{3}^{*}=C_{3}=A$, $A_{4}=B^{*}$, and $C_{4}=D$, we can easily get the proof.

In Theorem 10, let $A_{1}, C_{1}, B_{1}$, and $C_{2}$ vanish. Then we can easily get the following.

Corollary 11. The extreme ranks of $S_{A}$ given by (42) with respect to $A^{\sim}$ are the following:

$$
\begin{aligned}
& \max _{A^{\sim}} r\left(S_{A}\right)=\min \left\{r\left[\begin{array}{ll}
D & B^{*}
\end{array}\right], r\left[\begin{array}{ccc}
0 & B & A \\
B^{*} & D & 0 \\
A & 0 & -A
\end{array}\right]-2 r(A)\right\}, \\
& \min _{A^{\sim}} r\left(S_{A}\right)=2 r\left[\begin{array}{ll}
D & B^{*}
\end{array}\right]+r\left[\begin{array}{ccc}
0 & B & A \\
B^{*} & D & 0 \\
A & 0 & -A
\end{array}\right]-2 r\left[\begin{array}{cc}
0 & B \\
B^{*} & D \\
A & 0
\end{array}\right] .
\end{aligned}
$$

\section{The Rank Invariance of (9)}

As another application of Theorem 4, we in this section consider the rank invariance of the matrix expression (9) with respect to the Hermitian solution of system (10).

Theorem 12. Suppose that (10) have Hermitian solution; then the rank of $f(X)$ defined by (9) with respect to the Hermitian solution of (10) is invariant if and only if

$$
\begin{gathered}
{\left[\begin{array}{cccc}
0 & A_{4}^{*} & B_{1} & A_{1}^{*} \\
A_{4} & C_{4} & 0 & 0 \\
A_{3} & 0 & -A_{3} C_{2} & -A_{3} C_{1}^{*} \\
B_{1}^{*} & 0 & -C_{2}^{*} B_{1} & -C_{2}^{*} A_{1}^{*} \\
A_{1} & 0 & -C_{1} B_{1} & -C_{1} A_{1}^{*}
\end{array}\right]} \\
=r\left[\begin{array}{cc}
C_{4} & A_{4} \\
C_{2}^{*} A_{4}^{*} & B_{1}^{*} \\
C_{1} A_{4}^{*} & A_{1}
\end{array}\right]+r\left[\begin{array}{l}
A_{3} \\
B_{1}^{*} \\
A_{1}
\end{array}\right],
\end{gathered}
$$$$
r\left[\begin{array}{ccccc}
0 & A_{4}^{*} & A_{3}^{*} & B_{1} & A_{1}^{*} \\
A_{4} & C_{4} & 0 & 0 & 0 \\
A_{3} & 0 & -C_{3} & -A_{3} C_{2} & -A_{3} C_{1}^{*} \\
B_{1}^{*} & 0 & -C_{2}^{*} A_{3}^{*} & -C_{2}^{*} B_{1} & -C_{2}^{*} A_{1}^{*} \\
A_{1} & 0 & -C_{1} A_{3}^{*} & -C_{1} B_{1} & -C_{1} A_{1}^{*}
\end{array}\right]+r\left[\begin{array}{l}
B_{1}^{*} \\
A_{1}
\end{array}\right]
$$

$$
=r\left[\begin{array}{cccc}
0 & A_{4}^{*} & B_{1} & A_{1}^{*} \\
A_{4} & C_{4} & 0 & 0 \\
A_{3} & 0 & -A_{3} C_{2} & -A_{3} C_{1}^{*} \\
B_{1}^{*} & 0 & -C_{2}^{*} B_{1} & -C_{2}^{*} A_{1}^{*} \\
A_{1} & 0 & -C_{1} B_{1} & -C_{1} A_{1}^{*}
\end{array}\right]+r\left[\begin{array}{c}
A_{3} \\
B_{1}^{*} \\
A_{1}
\end{array}\right]
$$

or

$$
\begin{gathered}
r\left[\begin{array}{cccc}
0 & A_{4}^{*} & B_{1} & A_{1}^{*} \\
A_{4} & C_{4} & 0 & 0 \\
A_{3} & 0 & -A_{3} C_{2} & -A_{3} C_{1}^{*} \\
B_{1}^{*} & 0 & -C_{2}^{*} B_{1} & -C_{2}^{*} A_{1}^{*} \\
A_{1} & 0 & -C_{1} B_{1} & -C_{1} A_{1}^{*}
\end{array}\right] \\
=r\left[\begin{array}{cc}
C_{4} & A_{4} \\
C_{2}^{*} A_{4}^{*} & B_{1}^{*} \\
C_{1} A_{4}^{*} & A_{1}
\end{array}\right]+r\left[\begin{array}{c}
A_{3} \\
B_{1}^{*} \\
A_{1}
\end{array}\right] .
\end{gathered}
$$

Proof. It is obvious that the rank of $f(X)$ with respect to Hermitian solution of system (10) is invariant if and only if

$$
\max r[f(X)]-\min r[f(X)]=0 .
$$

By (49), Theorem 4, and simplifications, we can get (47) and (48). 
Corollary 13. The rank of $S_{A}$ defined by (42) with respect to $A^{\sim}$ which is a solution to system (2) is invariant if and only if

$$
r\left[\begin{array}{cccc}
0 & B & B_{1} & A_{1}^{*} \\
B^{*} & D & 0 & 0 \\
A & 0 & -A C_{2} & -A C_{1}^{*} \\
B_{1}^{*} & 0 & -C_{2}^{*} B_{1} & -C_{2}^{*} A_{1}^{*} \\
A_{1} & 0 & -C_{1} B_{1} & -C_{1} A_{1}^{*}
\end{array}\right]=r\left[\begin{array}{cc}
D & B^{*} \\
C_{2}^{*} B & B_{1}^{*} \\
C_{1} B & A_{1}
\end{array}\right]+r\left[\begin{array}{c}
A \\
B_{1}^{*} \\
A_{1}
\end{array}\right],
$$$$
r\left[\begin{array}{ccccc}
0 & B & A & B_{1} & A_{1}^{*} \\
B^{*} & D & 0 & 0 & 0 \\
A & 0 & -A & -A C_{2} & -A C_{1}^{*} \\
B_{1}^{*} & 0 & -C_{2}^{*} A & -C_{2}^{*} B_{1} & -C_{2}^{*} A_{1}^{*} \\
A_{1} & 0 & -C_{1} A & -C_{1} B_{1} & -C_{1} A_{1}^{*}
\end{array}\right]+r\left[\begin{array}{c}
B_{1}^{*} \\
A_{1}
\end{array}\right]
$$$$
=r\left[\begin{array}{cccc}
0 & B & B_{1} & A_{1}^{*} \\
B^{*} & D & 0 & 0 \\
A & 0 & -A C_{2} & -A C_{1}^{*} \\
B_{1}^{*} & 0 & -C_{2}^{*} B_{1} & -C_{2}^{*} A_{1}^{*} \\
A_{1} & 0 & -C_{1} B_{1} & -C_{1} A_{1}^{*}
\end{array}\right]+r\left[\begin{array}{c}
A \\
B_{1}^{*} \\
A_{1}
\end{array}\right]
$$

or

$$
\begin{gathered}
{\left[\begin{array}{cccc}
0 & B & B_{1} & A_{1}^{*} \\
B^{*} & D & 0 & 0 \\
A & 0 & -A C_{2} & -A C_{1}^{*} \\
B_{1}^{*} & 0 & -C_{2}^{*} B_{1} & -C_{2}^{*} A_{1}^{*} \\
A_{1} & 0 & -C_{1} B_{1} & -C_{1} A_{1}^{*}
\end{array}\right]} \\
=r\left[\begin{array}{cc}
D & B^{*} \\
C_{2}^{*} B & B_{1}^{*} \\
C_{1} B & A_{1}
\end{array}\right]+r\left[\begin{array}{c}
A \\
B_{1}^{*} \\
A_{1}
\end{array}\right] .
\end{gathered}
$$

\section{Acknowledgments}

This research was supported by the National Natural Science Foundation of China, Tian Yuan Foundation (11226067), the Fundamental Research Funds for the Central Universities (WM1214063), and China Postdoctoral Science Foundation (2012M511014).

\section{References}

[1] T. W. Hungerford, Algebra, Springer, New York, NY, USA, 1980.

[2] S. K. Mitra, "The matrix equations $A X=C, X B=D$," Linear Algebra and its Applications, vol. 59, pp. 171-181, 1984.

[3] C. G. Khatri and S. K. Mitra, "Hermitian and nonnegative definite solutions of linear matrix equations," SIAM Journal on Applied Mathematics, vol. 31, no. 4, pp. 579-585, 1976.

[4] Y. X. Yuan, "On the symmetric solutions of matrix equation $(A X, X C)=(B, D)$," Journal of East China Shipbuilding Institute, vol. 15, no. 4, pp. 82-85, 2001 (Chinese).

[5] H. Dai and P. Lancaster, "Linear matrix equations from an inverse problem of vibration theory," Linear Algebra and its Applications, vol. 246, pp. 31-47, 1996.
[6] J. Groß, "A note on the general Hermitian solution to $A X A^{*}=$ $B$," Malaysian Mathematical Society, vol. 21, no. 2, pp. 57-62, 1998.

[7] Y. G. Tian and Y. H. Liu, "Extremal ranks of some symmetric matrix expressions with applications," SIAM Journal on Matrix Analysis and Applications, vol. 28, no. 3, pp. 890-905, 2006.

[8] Y. H. Liu, Y. G. Tian, and Y. Takane, "Ranks of Hermitian and skew-Hermitian solutions to the matrix equation $A X A^{*}=B$," Linear Algebra and its Applications, vol. 431, no. 12, pp. 2359$2372,2009$.

[9] X. W. Chang and J. S. Wang, "The symmetric solution of the matrix equations $A X+Y A=C, A X A^{T}+B Y B^{T}=C$ and $\left(A^{T} X A, B^{T} X B\right)=(C, D)$," Linear Algebra and its Applications, vol. 179, pp. 171-189, 1993 .

[10] Q.-W. Wang and Z.-C. Wu, "Common Hermitian solutions to some operator equations on Hilbert $C^{*}$-modules," Linear Algebra and its Applications, vol. 432, no. 12, pp. 3159-3171, 2010.

[11] F. O. Farid, M. S. Moslehian, Q.-W. Wang, and Z.-C. Wu, "On the Hermitian solutions to a system of adjointable operator equations," Linear Algebra and its Applications, vol. 437, no. 7, pp. 1854-1891, 2012.

[12] Z.-H. He and Q.-W. Wang, "Solutions to optimization problems on ranks and inertias of a matrix function with applications," Applied Mathematics and Computation, vol. 219, no. 6, pp. 29893001, 2012.

[13] Q.-W. Wang, "The general solution to a system of real quaternion matrix equations," Computers \& Mathematics with Applications, vol. 49, no. 5-6, pp. 665-675, 2005.

[14] Q.-W. Wang, "Bisymmetric and centrosymmetric solutions to systems of real quaternion matrix equations," Computers \& Mathematics with Applications, vol. 49, no. 5-6, pp. 641-650, 2005.

[15] Q. W. Wang, "A system of four matrix equations over von Neumann regular rings and its applications," Acta Mathematica Sinica, vol. 21, no. 2, pp. 323-334, 2005.

[16] Q.-W. Wang, "A system of matrix equations and a linear matrix equation over arbitrary regular rings with identity," Linear Algebra and its Applications, vol. 384, pp. 43-54, 2004.

[17] Q.-W. Wang, H.-X. Chang, and Q. Ning, "The common solution to six quaternion matrix equations with applications," Applied Mathematics and Computation, vol. 198, no. 1, pp. 209-226, 2008.

[18] Q.-W. Wang, H.-X. Chang, and C.-Y. Lin, "P-(skew)symmetric common solutions to a pair of quaternion matrix equations," Applied Mathematics and Computation, vol. 195, no. 2, pp. 721732, 2008.

[19] Q. W. Wang and Z. H. He, "Some matrix equations with applications," Linear and Multilinear Algebra, vol. 60, no. 11-12, pp. 1327-1353, 2012.

[20] Q. W. Wang and J. Jiang, "Extreme ranks of (skew-)Hermitian solutions to a quaternion matrix equation," Electronic Journal of Linear Algebra, vol. 20, pp. 552-573, 2010.

[21] Q.-W. Wang and C.-K. Li, "Ranks and the least-norm of the general solution to a system of quaternion matrix equations," Linear Algebra and its Applications, vol. 430, no. 5-6, pp. 1626$1640,2009$.

[22] Q.-W. Wang, X. Liu, and S.-W. Yu, "The common bisymmetric nonnegative definite solutions with extreme ranks and inertias to a pair of matrix equations," Applied Mathematics and Computation, vol. 218, no. 6, pp. 2761-2771, 2011. 
[23] Q.-W. Wang, F. Qin, and C.-Y. Lin, "The common solution to matrix equations over a regular ring with applications," Indian Journal of Pure and Applied Mathematics, vol. 36, no. 12, pp. 655672, 2005.

[24] Q.-W. Wang, G.-J. Song, and C.-Y. Lin, "Extreme ranks of the solution to a consistent system of linear quaternion matrix equations with an application," Applied Mathematics and Computation, vol. 189, no. 2, pp. 1517-1532, 2007.

[25] Q.-W. Wang, G.-J. Song, and C.-Y. Lin, "Rank equalities related to the generalized inverse $A_{T, S}^{(2)}$ with applications," Applied Mathematics and Computation, vol. 205, no. 1, pp. 370-382, 2008.

[26] Q. W. Wang, G. J. Song, and X. Liu, "Maximal and minimal ranks of the common solution of some linear matrix equations over an arbitrary division ring with applications," Algebra Colloquium, vol. 16, no. 2, pp. 293-308, 2009.

[27] Q.-W. Wang, Z.-C. Wu, and C.-Y. Lin, "Extremal ranks of a quaternion matrix expression subject to consistent systems of quaternion matrix equations with applications," Applied Mathematics and Computation, vol. 182, no. 2, pp. 1755-1764, 2006.

[28] Q. W. Wang and S. W. Yu, "Ranks of the common solution to some quaternion matrix equations with applications," Bulletin of Iranian Mathematical Society, vol. 38, no. 1, pp. 131-157, 2012.

[29] Q. W. Wang, S. W. Yu, and W. Xie, "Extreme ranks of real matrices in solution of the quaternion matrix equation $A X B=$ $C$ with applications," Algebra Colloquium, vol. 17, no. 2, pp. 345360, 2010.

[30] Q.-W. Wang, S.-W. Yu, and Q. Zhang, "The real solutions to a system of quaternion matrix equations with applications," Communications in Algebra, vol. 37, no. 6, pp. 2060-2079, 2009.

[31] Q.-W. Wang, S.-W. Yu, and C.-Y. Lin, "Extreme ranks of a linear quaternion matrix expression subject to triple quaternion matrix equations with applications," Applied Mathematics and Computation, vol. 195, no. 2, pp. 733-744, 2008.

[32] Q.-W. Wang and F. Zhang, "The reflexive re-nonnegative definite solution to a quaternion matrix equation," Electronic Journal of Linear Algebra, vol. 17, pp. 88-101, 2008.

[33] Q. W. Wang, X. Zhang, and Z. H. He, "On the Hermitian structures of the solution to a pair of matrix equations," Linear and Multilinear Algebra, vol. 61, no. 1, pp. 73-90, 2013.

[34] X. Zhang, Q.-W. Wang, and X. Liu, "Inertias and ranks of some Hermitian matrix functions with applications," Central European Journal of Mathematics, vol. 10, no. 1, pp. 329-351, 2012.

[35] Y. G. Tian and S. Z. Cheng, "The maximal and minimal ranks of A - BXC with applications," New York Journal of Mathematics, vol. 9, pp. 345-362, 2003.

[36] Y. G. Tian, "Upper and lower bounds for ranks of matrix expressions using generalized inverses," Linear Algebra and its Applications, vol. 355, pp. 187-214, 2002.

[37] Y. H. Liu and Y. G. Tian, "More on extremal ranks of the matrix expressions $A-B X \pm X^{*} B^{*}$ with statistical applications," Numerical Linear Algebra with Applications, vol. 15, no. 4, pp. 307-325, 2008.

[38] Y. H. Liu and Y. G. Tian, "Max-min problems on the ranks and inertias of the matrix expressions $A-B X C \pm(B X C)^{*}$ with applications," Journal of Optimization Theory and Applications, vol. 148, no. 3, pp. 593-622, 2011.

[39] T. Ando, "Generalized Schur complements," Linear Algebra and its Applications, vol. 27, pp. 173-186, 1979.
[40] D. Carlson, E. Haynsworth, and T. Markham, "A generalization of the Schur complement by means of the Moore-Penrose inverse," SIAM Journal on Applied Mathematics, vol. 26, pp. 169$175,1974$.

[41] M. Fiedler, "Remarks on the Schur complement," Linear Algebra and its Applications, vol. 39, pp. 189-195, 1981.

[42] Y. G. Tian, "More on maximal and minimal ranks of Schur complements with applications," Applied Mathematics and Computation, vol. 152, no. 3, pp. 675-692, 2004. 


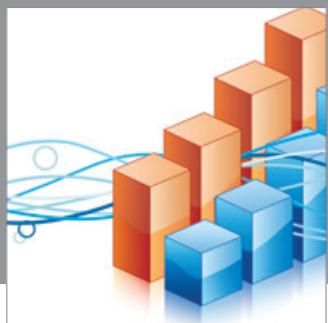

Advances in

Operations Research

mansans

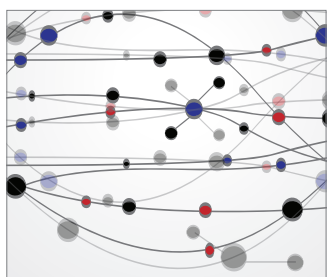

The Scientific World Journal
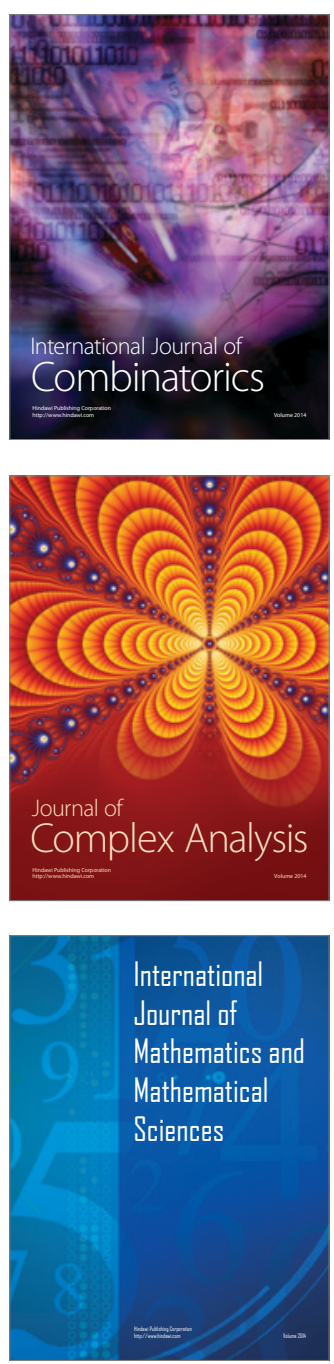
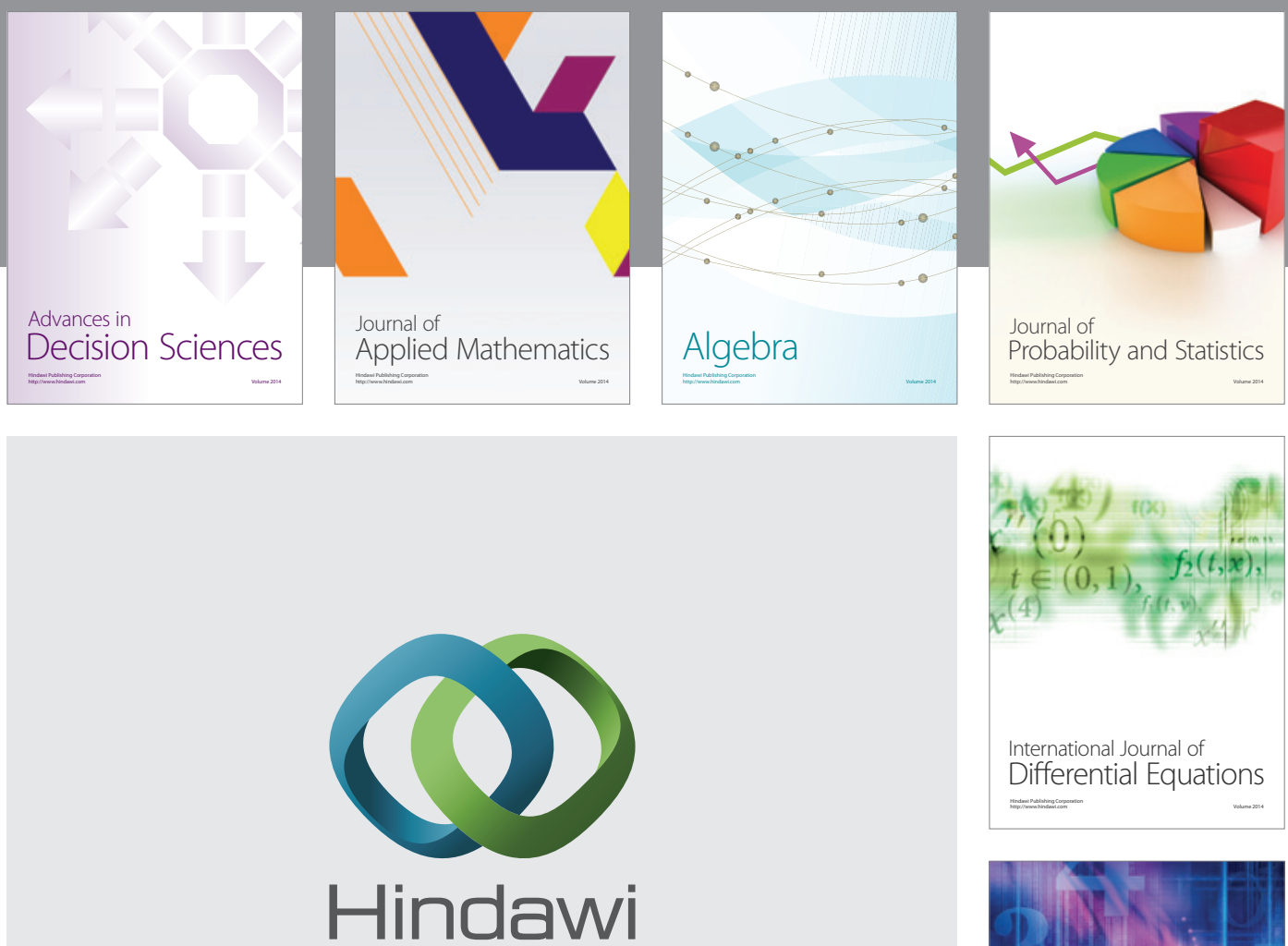

Submit your manuscripts at http://www.hindawi.com
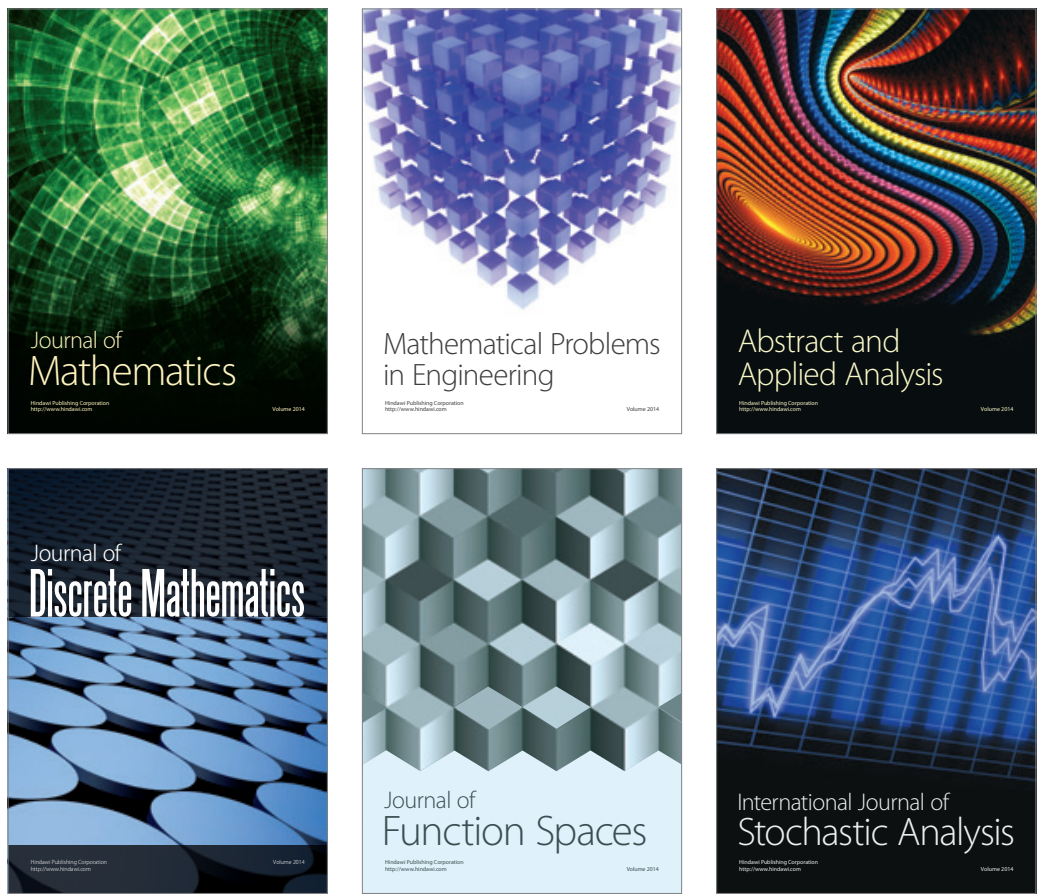

Journal of

Function Spaces

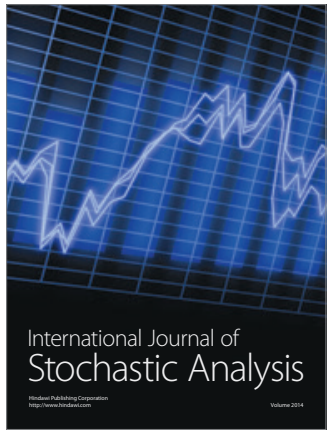

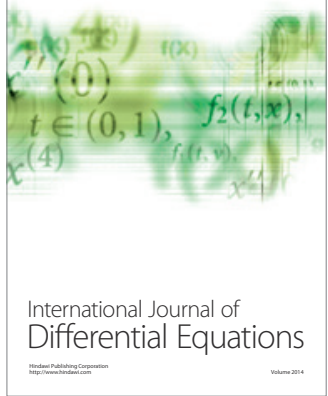
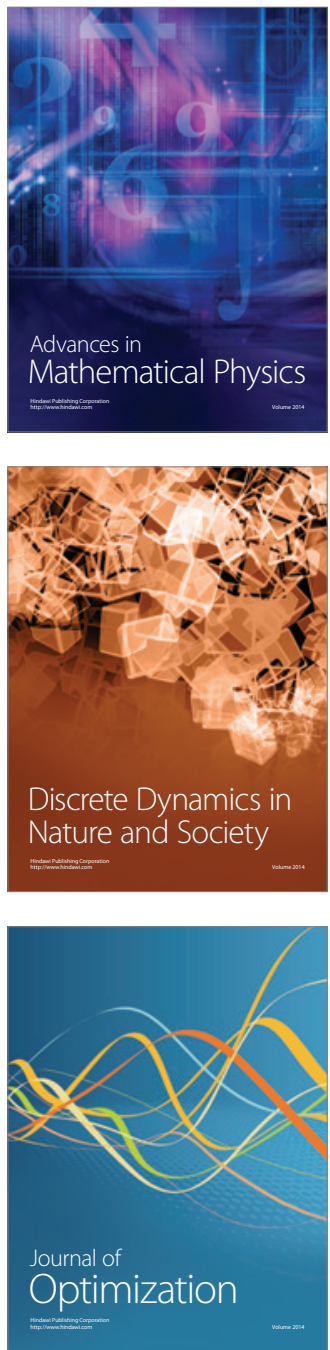